\title{
The Nature, Cause and Consequence of COVID-19 Panic among Social Media Users in India
}

Chandrima Bhattacharya, $\mathrm{MS}^{1}$, Debaroti Chowdhury, Senior ${ }^{2}$, Nova Ahmed, $\mathrm{PhD}^{3}$, Su Özgür, $\mathrm{PhD}^{4}$, Bodhisatwa Bhattacharya, Senior ${ }^{5}$,

Sankar Kumar Mridha, $\mathrm{MTech}^{6}$, and Malay Bhattacharyya, $\mathrm{PhD}^{7,8, *}$

${ }^{1}$ The HRH Prince Alwaleed Bin Talal Bin Abdulaziz Alsaud

Institute for Computational Biomedicine,

Weill Cornell Medicine, New York, NY, 10065, USA

${ }^{2}$ Department of Computer Science and Technology

Indian Institute of Engineering Science and Technology, Shibpur

West Bengal - 711103, India

${ }^{3}$ Department of Electrical and Computer Engineering

North South University

Bashundhara, Dhaka - 1229, Bangladesh

${ }^{4}$ Department of Biostatistics and Medical Informatics

Ege University Faculty of Medicine

Bornova 35100, İzmir, Turkey

${ }^{5}$ Calcutta Boys' School

Kolkata, West Bangal - 700014, India

${ }^{6}$ Department of Information Technology

Indian Institute of Engineering Science and Technology, Shibpur

West Bengal - 711103, India

${ }^{7}$ Centre for Artificial Intelligence and Machine Learning

Indian Statistical Institute, Kolkata

West Bengal - 700108, India

${ }^{8}$ Machine Intelligence Unit

Indian Statistical Institute, Kolkata

West Bengal - 700108, India

Word count of the manuscript text: 3900 words

*Correspondence should be addressed to malaybhattacharyya@isical.ac.in. 


\begin{abstract}
Aims

The recent pandemic of COVID-19 has not only shaken the healthcare but also economic structure around the world. In addition to these direct effects, it has also brought in some indirect difficulties owing to the information epidemic on social media. As India experienced a later outbreak of COVID-19 and a prolonged uninterrupted lockdown, we aimed to understand the nature of panic social media users in India are experiencing due to the flow of (mis)information. We further extend this investigation to other countries.

\section{Methods}

We performed a cross-sectional study by conducting survey on multiple social media platforms. We received 1075 responses (sex ratio 2:1) through opportunity sampling from social media users of 30 different countries (between April 11, 2020 and May 15, 2020). We performed both quantitative and qualitative analyses on the 935 respondents from India. Several hypotheses are statistically tested on them and are further examined on rest of the 140 social media users from 29 other countries. We also performed a separate Twitter hashtag analysis and sentiment analysis on the responses. We applied a citizen science approach to involve the respondents in the analysis pipeline after the survey.

\section{Results}

This cross-sectional study on 1075 social media users from India and 29 other countries revealed a significant increase of social media usage and rise of panic over time in India. Middle-aged people and female exhibit a higher panic in India. The amount of panic was independent of the nature of association with COVID-19. The change of mental health was associated with panic level and productivity. Further qualitative analysis highlights the occurrences of information panic, economic panic, moral panic and spiritual panic, among other causes.

\section{Conclusions}

Several panic behaviors are unique to social media users in India possibly because COVID-19 broke out relatively later in comparison with the other countries and the uninterrupted lockdown prolonged for a long time. The amount of social media usage might not be causal but has a significant role in generating panic among the people in India. A significantly higher level of panic among the middle-aged people can be attributed to their higher amount of responsibility. The popularity of different hashtags, including the names of drugs under trial for COVID-19, in limited countries highlight that the causes of panic are not the same everywhere. As some of the respondents took part as citizen scientists a robust perspective to the outcome is obtained.
\end{abstract}

Keywords: COVID-19, panic, anxiety, mental health, social media. 


\section{Introduction}

The COVID-19 pandemic has caused direct losses like the collapse of public healthcare support and economic conditions around the world. In addition to this, mental health of people has been deeply affected by the prolonged change of social and economic activities of people. COVID-19 has globally affected the mental health of people in a significant way due to the incidents like economic slowdown, loss of jobs, losing the loved ones, and so on. However, there are other hidden factors causing the panic and anxiety among people. Containment methods (e.g., lockdown, travel restrictions, social distancing, etc.) appear to be the main anti-contagion policies to fight against COVID-19 pandemic throughout the world [14]. This has caused a major impact on the mental health of people. The focus on clinical health has been enormous, but the necessary psychological health protocols have not been established for an event like COVID-19 pandemic. Hence, there have been reports of people breaking the rules around the world. As a matter of fact, there is hardly any Psychological First Aid available for confronting COVID-19 in India.

As social media has emerged as a major source of knowledge and communication, there is a (mis)information epidemic of COVID-19 on various online social platforms [10, 22]. It is therefore interesting to study whether we are experiencing the panic of COVID-19 pandemic or pandemic of COVID-19 panic on social media [27]. Recent studies highlight that the spread of fake information is creating considerable panic among the people in several countries [15]. Fig. 1 shows an example of fake information being spread in Facebook during the COVID-19 lockdown imposed in India. Misleading information and conspiracy theories has been an integral part of panic, and social media just adds the right medium for propagation and spread of panic. There has been a steep rise of moral panic [4] which was observed during multiple events earlier. Hence, it is highly likely that COVID-19 will also cause moral panic and catalyze the spread of fear through social media leading to a panic pandemic of its own.
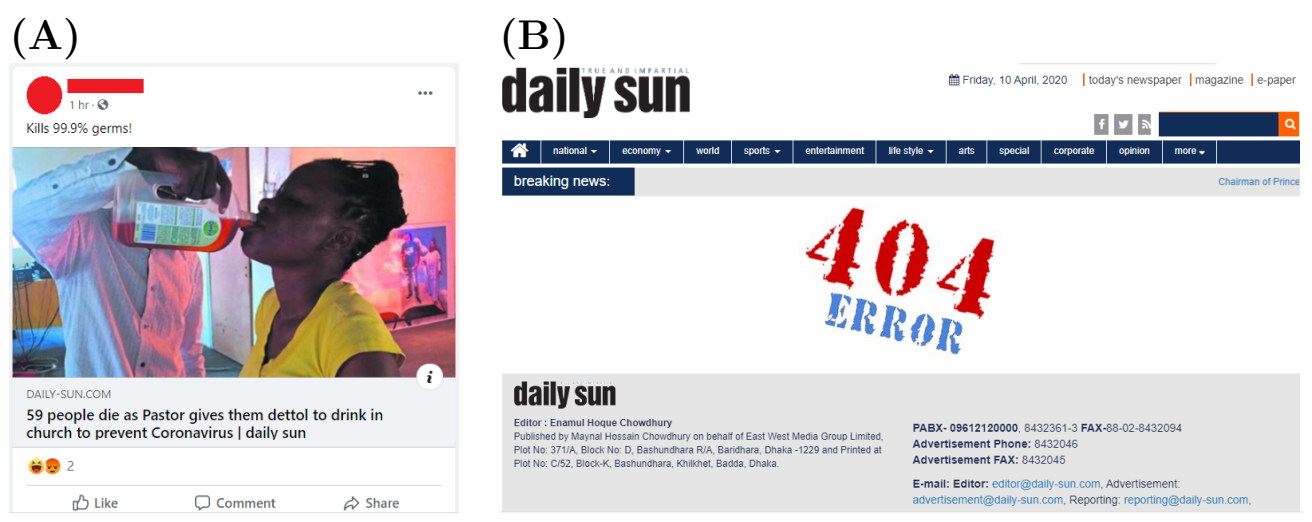

Figure 1: Spread of COVID-19 misinformation on social media. (A) Information related to COVID-19 with hyperlinks are getting spread on social media. (B) Many such information are fake in nature and lead to nowhere.

Although social media plays a major role in spreading misinformation, however, all the topics are not discussed with equal priority in each region around the world. Fig. 2 reflects that the popularity of the topic hydroxychloriquine, a controversial drug under trial for 
COVID-19 [11, 17, 21], on Twitter is restricted to limited number of countries. This is possibly because the expectations and resulting panic out of this drug is not the same everywhere. Hence, a region specific analysis is necessary for appropriately understanding the amount of COVID-19 panic among a group of social media users. As India experienced a prolonged and uninterrupted lockdown since March 25, 2020, performing a panic analysis on the Indian population could be compelling. With this motivation, the current study aims to realize the nature, causes and consequences of panic among the social media users in India.

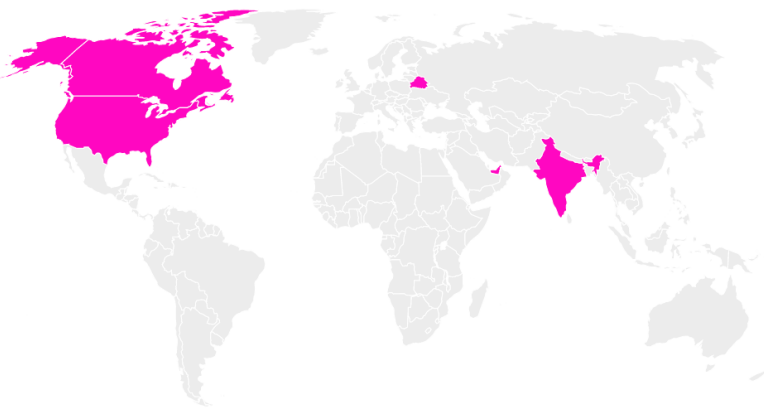

Figure 2: Countrywise popularity of the Twitter hashtag \#hydroxychloriquine. Countries in which the hashtag \#hydroxychloriquine appeared at least once in the top 50 list on Twitter during the COVID-19 pandemic. The data is collected until May 18, 2020.

\section{Related Work}

Recent studies have highlighted that multiple types of psychological trauma follow COVID19 pandemic, including fear of death, physical illness due to decreased immunity in patients, economic downfall curbed with loss of employment [24], anxiety [28], depression, stress, insomnia, boredom, and loneliness from prolonged quarantine phases [38].

Irrespective of social media being the source of self-gratitude by news-sharing [18], multiple studies have shown that the same platform can be used in to spread misinformation $[23,31]$, thus swaying the mindset of people and indirectly inducing psychological traumas as seen by past events $[2,35,36,37]$. Multiple studies focusing on mitigating misinformation has shown that might be a way of enhancing the quality of mental health during the pandemic $[5,6,9]$.

WHO has published guidelines to be followed for mental health and well-being during COVID-19 pandemic [25]. Most countries have developed multiple forms of mental health mitigation to cope with neuropsychriatric linkage associated with phased of COVID-19 [26, 29]. Some studies also mention the necessity for using Psychological First Aid (PFA) to combat with the rising fear during any pandemic $[12,20]$.

Even with Government in different countries and WHO trying to mitigate panic and fear, behavior associated with fear including panic buying of essential commodities (including food, masks, hand sanitizer) has been seen across the world [19, 32]. There has also been a global trend of increase in suicidal thoughts caused by both fear of unemployment [16] as well as fear of unknown caused by the virus $[8,28]$. There is an immediate global need for 
assessment and understanding of associated behavior and distress signs including sleeping disorder and anxiety, to mitigate and reduce suicidal risks [30, 39].

\section{Data Details}

We received responses from 1075 social media users (median age group 21-30 years, 33.1\% female) from 30 different countries between April 11, 2020 and May 15, 2020 (see Supplementary File 2). Most of the respondents were from Asia (about 91\%). The other demography related information of the respondents are summarized in Fig. 3. Most of the responding people were young, belonging either to the age group of 21-30 years $(58.7 \%)$ or below 21 years (14.2\%) (see Fig. 3). A majority of the respondents (57.5\%) had no direct association with COVID-19.

\section{Methods}

We performed a cross-sectional study on the users from multiple social media platforms. We collected survey responses by circulating Google Forms with questionnaire (see Supplementary File 1) through opportunity sampling via Facebook, WhatsApp, Twitter and LinkedIn. The data is segregated into two parts - the first one comprising 935 responses received from India and the other one with rest of the 140 responses. We performed both quantitative and qualitative analyses separately on the Indian social media users and on the ones in other 29 countries. The values received in the form of numeric intervals are either studied as categorical values (e.g., age group) or averaged to derive a single value (e.g., social media usage). We grouped respondents into five distinct buckets of equal sizes for conducting temporal analysis of the panic level acquired over time.

The Twitter data is collected using the GetDayTrends website ${ }^{1}$ on May 18, 2020. The COVID-19 related hashtags that appeared in the top 50 list at least once in India since the COVID-19 outbreak (in March, 2020) are chosen. The details of these hashtags like the appearance and rank are also taken.

The sentiment analysis results were obtained using the Magellan Text Mining tool ${ }^{2}$ of OpenText. It uses a built-in multilingual natural language processing approach for extracting topics, sentiments and entities. We collected the values of Topics (weight), Simple Context (Relevancy \%, Frequency), Complex Context (Relevancy \%, Frequency), Entities of Interest (Relevancy \%, Frequency) only when frequency of occurrence is no less than 2, Sentiment Divisions (Positive \%, Negative \%, Neutral \%), Comments Type (Opinion, Facts). Sentiment values were calculated using the Sentiment Analyzer tool ${ }^{3}$. The values lie in the range $[-100,100]$.

\footnotetext{
${ }^{1}$ https://getdaytrends.com

${ }^{2}$ http://magellan-text-mining.opentext.com

${ }^{3}$ https://www.danielsoper.com/sentimentanalysis/default.aspx
} 
(A)

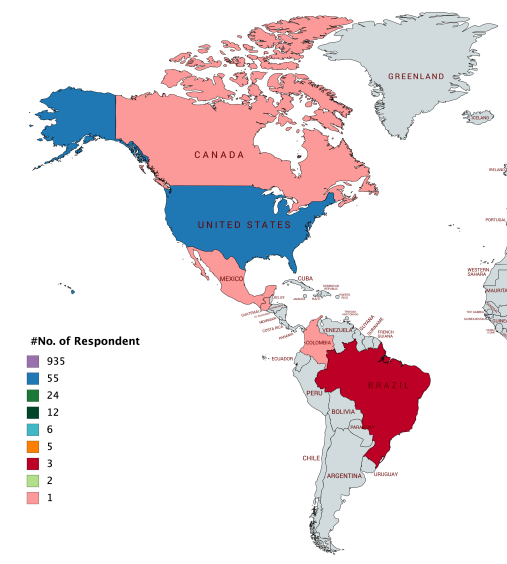

(B)

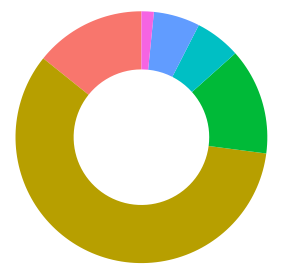

$(\mathrm{E})$

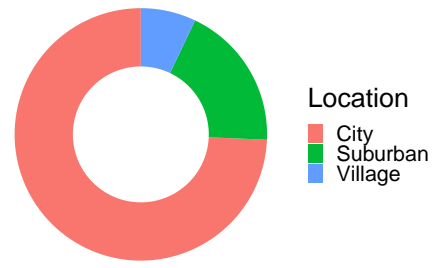

(C)
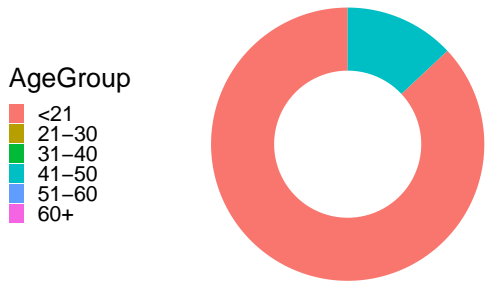

Country India

$(\mathrm{F})$

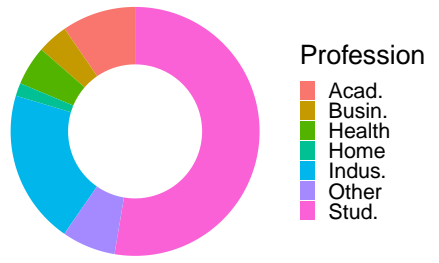

(D)

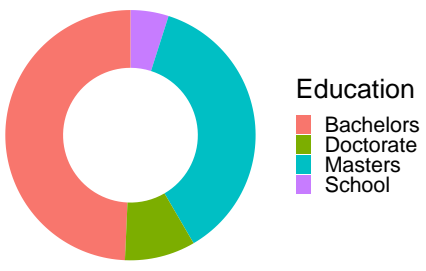

$(\mathrm{G})$

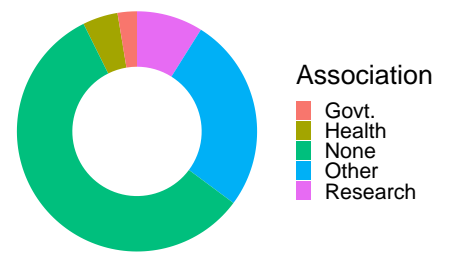

Figure 3: Demography and COVID-19 association of respondents. (A) Countries to which the 1075 people responding to the online survey during the COVID-19 pandemic belong across the world. (B-F) Demographic details of the respondents. (G) Nature of COVID-19 association of the respondents. 


\section{Results}

We found that the social media usage has significantly increased (McNemar Bowker, $p<$ 0.001) across all the countries during the lockdown (see Supplementary Details, Table S3). This is true even within India and within other countries taken in separation. We observed that the mean usage (calculated from averaged interval) of social media has become 2.25 hrs per day in India during lockdown, while it happened to be $1.66 \mathrm{hrs}$ per day earlier. The social media usage has significantly increased (Wilcoxon-Mann-Whitney, $p=2.2 \mathrm{e}-16$ ) during lockdown (see Fig. 4(A)). The panic levels in India and other countries do not follow normal distributions (see Supplementary Details, Table S4). No significant difference of panic level (Wilcoxon-Mann-Whitney, $p=0.5426$ ) is observed between India and other countries, although it exhibits a slightly higher value and variability of panic in India (see Fig. 4(B)). On grouping the respondents from India into equal sized cohorts based on the temporal order of their participation in the survey, we observed a significant increase of panic over time (see Fig. 4(C)). However, a similar analysis highlights no such increase in other countries (see Supplementary Details, Fig. S3). A possible reason of this could be the fact that COVID19 broke out relatively later in India in comparison with other countries and there was a prolonged and uninterrupted lockdown. The panic level in India is neither dependent on the usage of social media before lockdown nor during lockdown (see Fig. 4(D)-(E)). However, the panic level has no association with the rise of social media usage in India. We noticed that the level of panic is independent of the association of a person (as Government personnel, health professional or researcher) with COVID-19 in India (see Fig. 4(F)). Moreover, the panic level is also independent of the rise of social media usage in India (see Fig. 4(G)). A similar observation was also obtained for other countries (see Supplementary Details, Fig. S3).

We examined the level of panic against the demographic details of the respondents to understand the differentiating factors of panic in India. The violin plots in Fig. 5 highlight the sample distributions of panic score over different subgroups of demographic factors (among Indian respondents) along with a non-parametric test result to verify whether they originate from the same distribution. We noticed that mean panic level of the people belonging to the age group 31-40 is significantly higher (Kruskal-Wallis, $p=0.0013$ ) than the others in India. Despite a higher fatality rate of COVID-19 reported amidst the older population [7], panic is getting generated only among the middle-aged people. It is interesting to note that younger people are not less panicked than the others. We found that females express a significantly higher level of panic than males in India (Wilcoxon-Mann-Whitney, $p=0.00063$ ). The same is also significantly higher in other countries (see Supplementary Details, Fig. S4). Finally, we found that the level of panic is independent of the nature of COVID-19 association, education, location type or profession of the respondents in India.

To realize how do people accept social media posts on COVID-19, we used Alluvial diagrams for highlighting associations between the different factors that people consider before sharing a post. The four categorical dimensions from left to right in Fig. 6 are the worldwide responses to the questions "Do you like the jokes/memes about COVID-19 and share?", "If your friend sends an apparently serious message on COVID-19 and asks you to forward, what will you do?", "When are you more likely to believe a statement on COVID-19 in a post?", and "How do you generally make a decision about sharing a COVID-19 post that contains a link?", respectively. Fig. 6 highlights that most of the people in India consider 
(A)

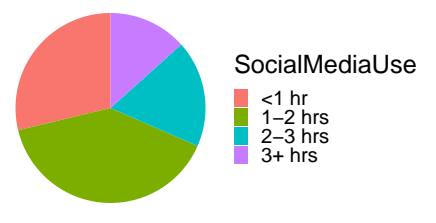

(D)

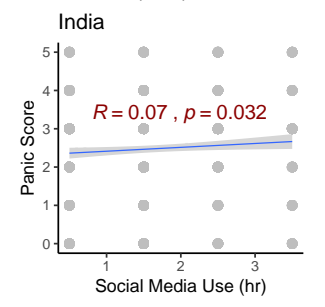

(B)

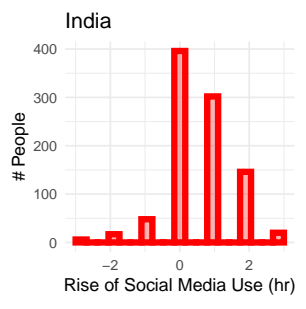

$(\mathrm{E})$

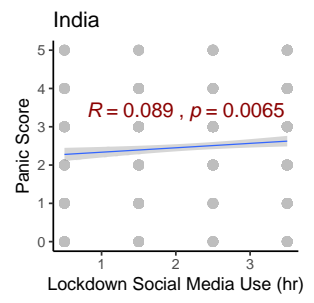

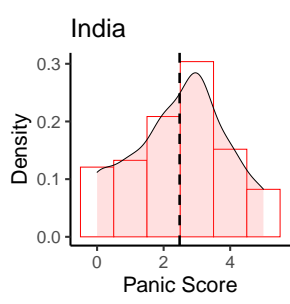

$(\mathrm{F})$

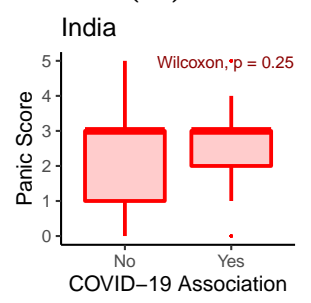

(C)

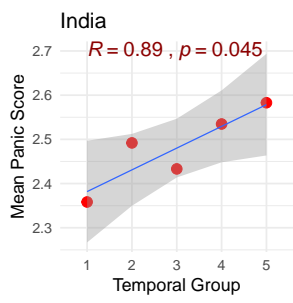

$(\mathrm{G})$

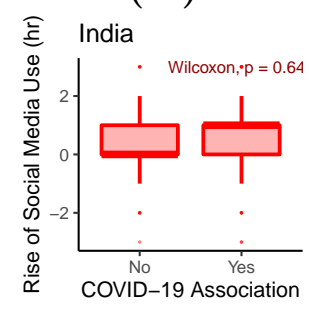

Figure 4: Effect of social media usage and COVID-19 association on the panic level in India. (A) The rise of social media usage during the lockdown. (B) Density of panic levels. (C) Mean panic level of people temporally grouped by the order of their participation in the survey. Correlations between the panic level and hourly usage of social media per day (D) before the lockdown and (E) during the lockdown. (F) Association with COVID-19 and its effect on the panic level. (G) Association with COVID-19 and its effect on the rise of social media usage during the lockdown. 
(A)

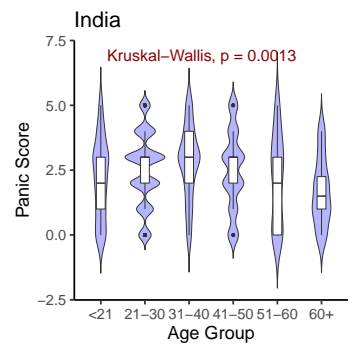

(D)

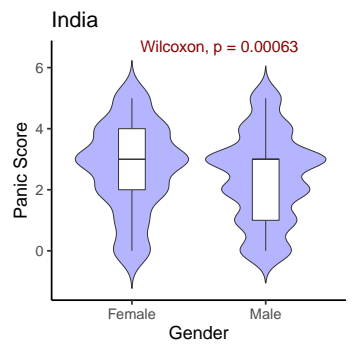

(B)

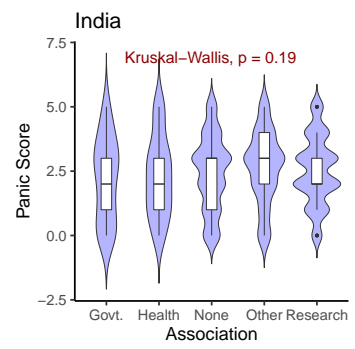

(E)

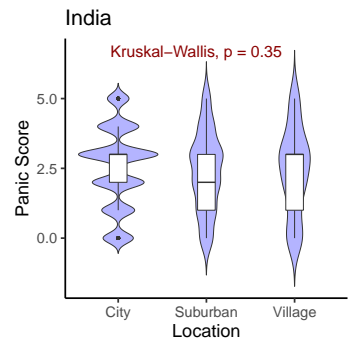

(C)

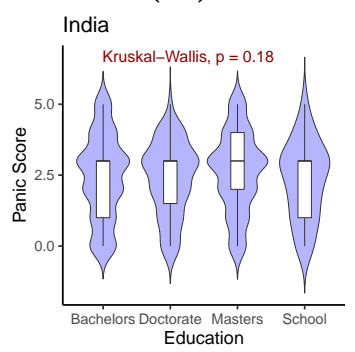

$(\mathrm{F})$

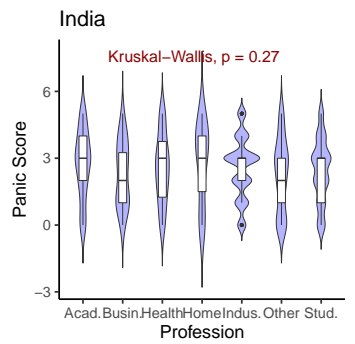

Figure 5: Panic level in India against different demographic factors. Violin plot showing the level of panic among different sets of people specific to their (A) age group has a significant difference, (B) association with COVID-19 has no significant difference, (C) level of education has no significant difference, (D) gender has a significant difference, (E) location type has no significant difference, and $(\mathrm{F})$ profession has no significant difference.

COVID-19 related posts seriously and share them only after validation. However, the nature of validation (verifying post content, associated link, and/or the linked document) differs among different sets of people.

Incomplete or lack of knowledge among social media users might be a reason of their anxiety. To better understand this, we studied how much people are aware about the disease COVID-19 and its different characteristics. We observed that a significant association exists between the understanding about COVID-19 and the profession of a person in India. In fact, the responses against the questions like what COVID-19 actually means (see Fig. 7(A)) or whether pneumonia vaccine can help prevent COVID-19 (see Fig. 7(B)) are significantly related to the profession (Chi-Square, $\chi^{2}=30.64, p=0.03$ and Chi-Square, $\chi^{2}=31.13$, $p=0.0019$, respectively). However, no such link is found for the people residing in the other countries (see Supplementary Details, Fig. S5). Further investigation highlights that the knowledge about COVID-19 is relatively poor among the common people in India in comparison with the other countries. To our surprise, a significantly higher number of respondents (test of proportions, $p=0.00052$ ) in India is found to believe that COVID-19 is a biological weapon. Such responses are received from $11 \%$ of the total samples in India, while from only $2 \%$ in other countries.

We further studied the various preventive activities undertaken by the respondents in India and other countries during lockdown (or other types of containment). These activities include the actions to take after returning from grocery, when a neighbor is tested COVID19 positive, and when someone you know is tested COVID-19 positive. We observed a 


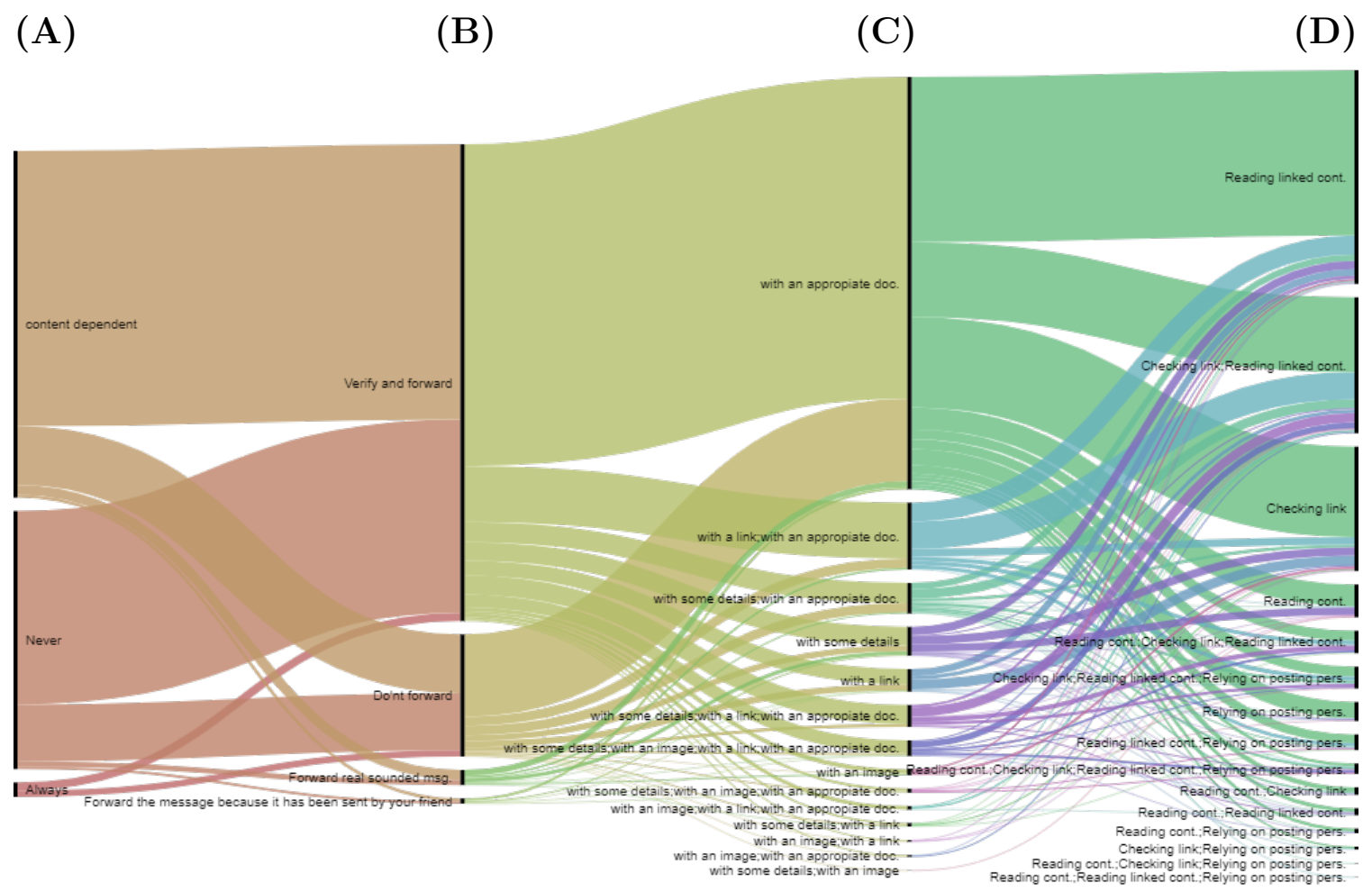

Figure 6: Sharing COVID-19 information on social media in India. Associations between the factors based on which people welcome and make decisions about sharing COVID19 related information on social media. The vertical bars cover responses to the questions on (A) liking and sharing jokes/memes about COVID-19, (B) forwarding an apparently serious message on COVID-19 sent by a friend, (C) believing a statement on COVID-19 in a post, and (D) making a decision about sharing a COVID-19 post that contains a link. 
(A)

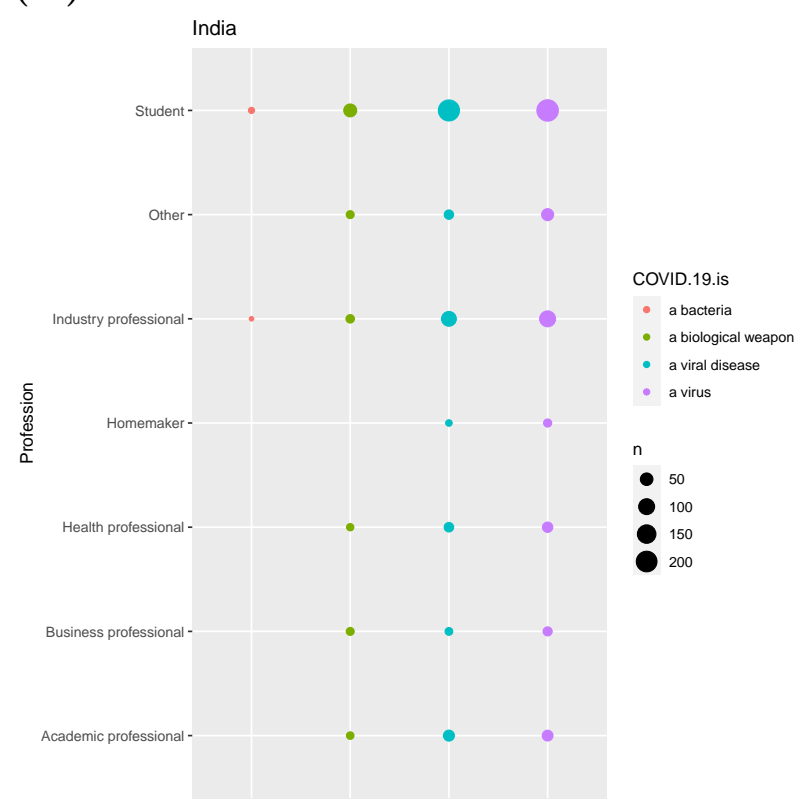

(B)

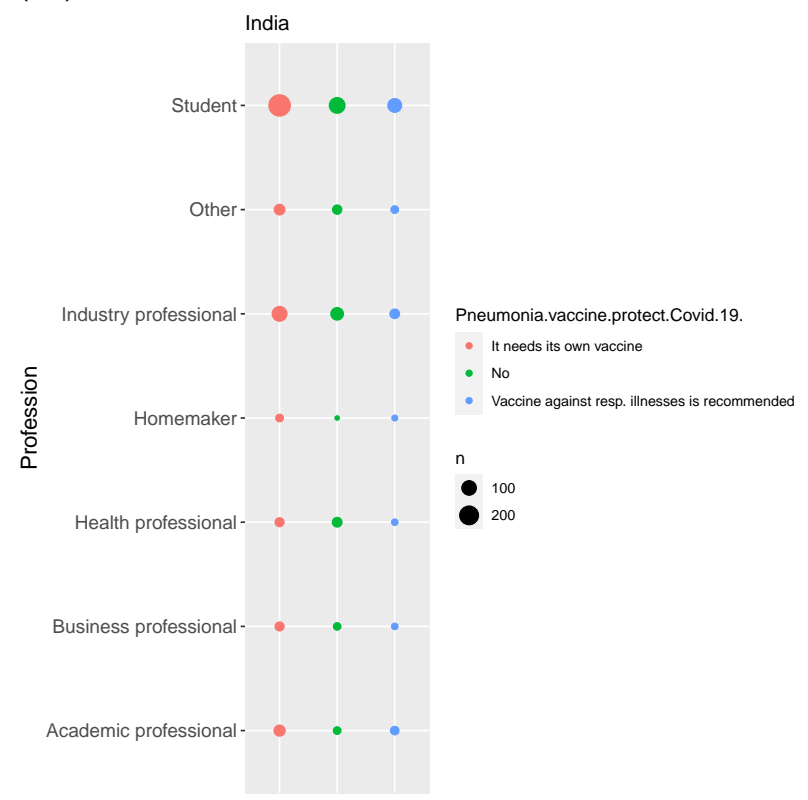

Figure 7: Association between the knowledge about COVID-19 and profession of the respondents in India. (A) Basic knowledge about COVID-19 is dependent on profession. (B) Basic knowledge about the treatment of COVID-19 is dependent on profession.

significant association between such activities and profession (Chi-Square, $\chi^{2}=113.46, p$ $=0.0178$, Chi-Square, $\chi^{2}=120.85, p=0.0053$, and Chi-Square, $\chi^{2}=113.46, p=0.0178$, respectively) of people in India but not in other countries (see Supplementary Details). A possible reason behind this could be the difference between government guidelines across the different countries. It is interesting to note that, a reasonable number of people $(7.2 \%)$ in India and rest of the countries (4.3\%) were found to be ready to consume hydroxychloroquine and azithromycin at their own in case they came into contact with a COVID-19 patient.

We examined the change of mental health (stress and anxiety) people experienced due to the lockdown protocols in their respective countries and how did it affect their level of panic. We observed a highly significant association (Chi-Square, $\chi^{2}=80.19, p=4.62 \mathrm{e}-13$ ) between the mental health and panic level in India (see Fig. 8(A)). We also noticed a highly significant association (Chi-Square, $\chi^{2}=42.40, p=1.38 \mathrm{e}-08$ ) between the mental health and productivity in India (see Fig. 8(B)). Both these associations were significant in other countries (see Supplementary Details, Fig. S6). We found a highly significant connection (see Fig. $8(\mathrm{C})$ ) between the mental health and level of tension felt after reading a post related to COVID-19 on social media in India (Chi-Square, $\chi^{2}=35.12, p=4.10 \mathrm{e}-06$ ) but not in other countries. We also observed a significant dependence (see Fig. 8(D)) between the method perceived by people to reduce panic level with their COVID-19 association in India (ChiSquare, $\chi^{2}=37.53, p=0.01$ ) but not in other countries. None of these associations were significant in other countries (see Supplementary Details, Fig. S6).

We verified whether the delay in getting a vaccine is responsible for the panic among people. We found that panic level is independent of the time expected (by the corresponding 
(A)

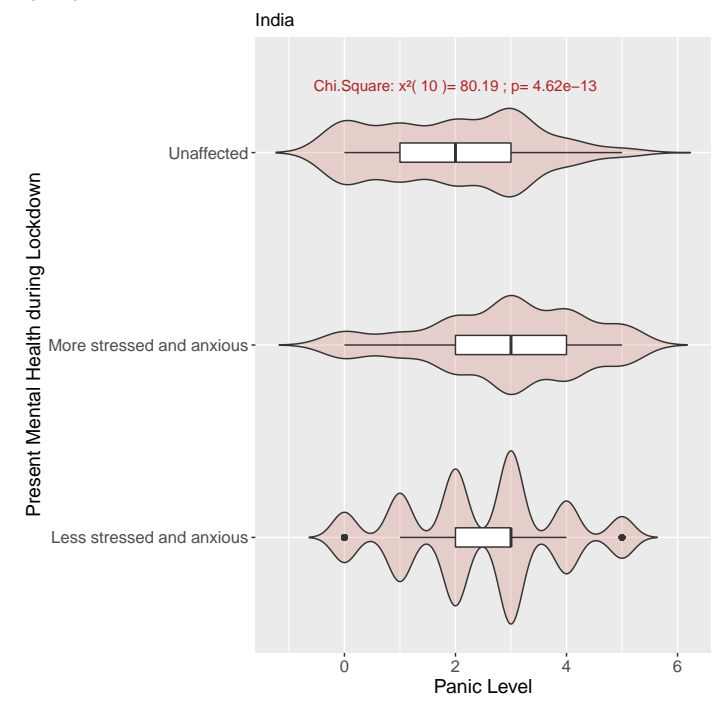

(C)

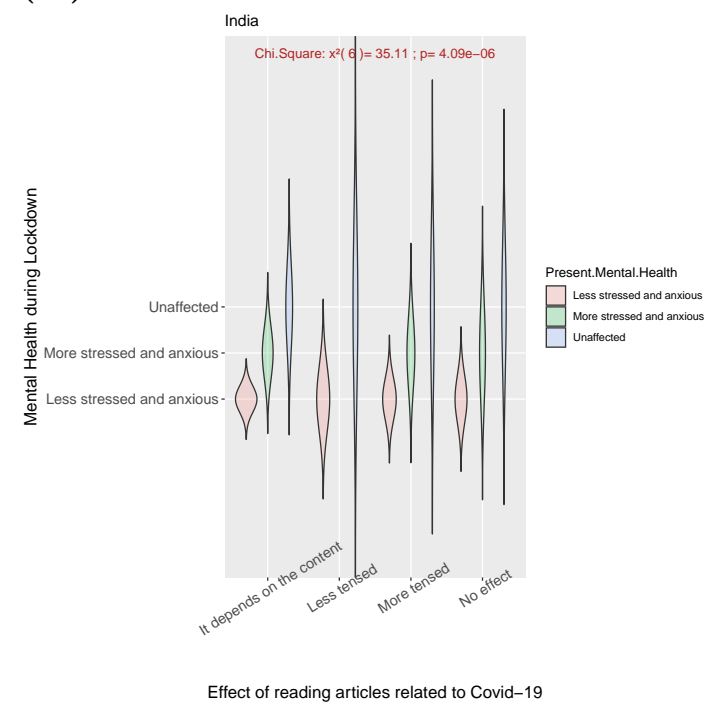

(B)

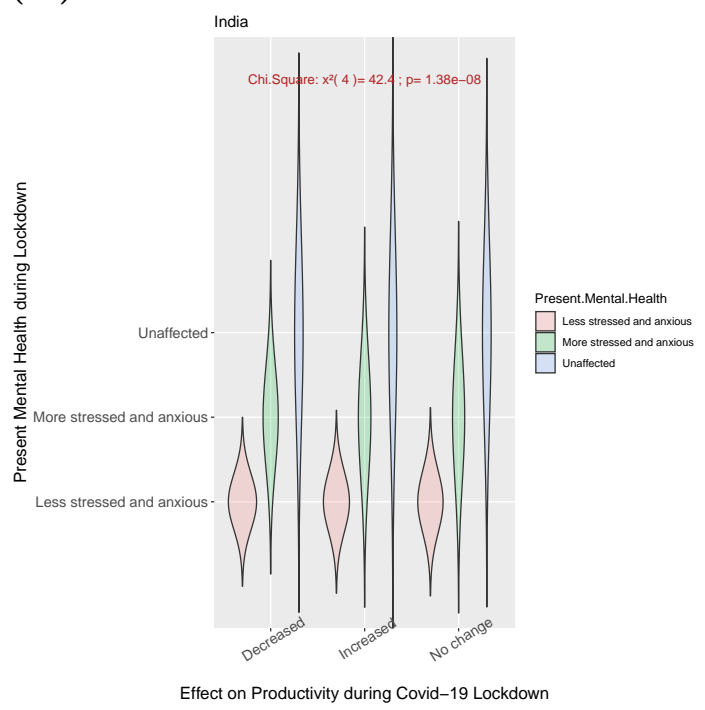

(D)

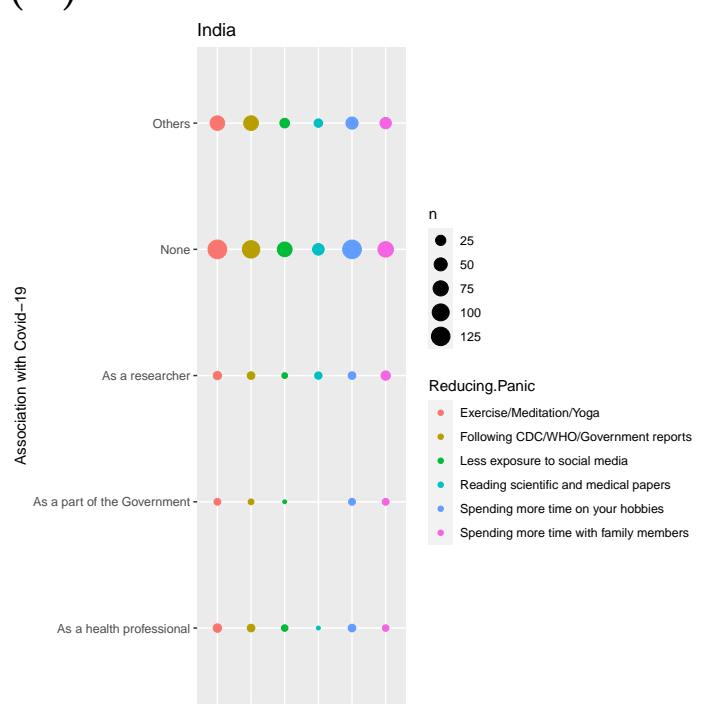

Figure 8: Association between mental health and other factors in India. (A) Violin plot depicting panic Level and its relation with mental health. (B) Violin plot depicting effect on productivity with respect to mental health, (C) Violin plot representing how much the tension felt after reading articles on COVID-19 affect mental health, (D) Ways to reduce panic as perceived by people associated to COVID-19 through various conditions. 
respondent) to get the vaccine in India (see Supplementary Details, Fig. S1). No significant dependence is also observed for other countries (see Supplementary Details, Fig. S7). We found no significant association between either the age group or profession of the respondents and their mental preparedness regarding the availability of COVID-19 vaccine either in India (see Supplementary Details, Fig. S2) or other countries (see Supplementary Details, Fig. S8). We noticed that the COVID-19 panic has a significant impact on the actions people take. We found a significant rise (Wilcoxon-Mann-Whitney, $p=0.0012$ ) of panic among the people who prefers to send younger people out for shopping just because aged ones are more prone to COVID-19 infection (see Supplementary Details).

On further studying the precautions taken by people in India based on their perceptions about COVID-19 (see Fig. 9), we found most of them (59\%) to be conditionally interested in a free test. This is also true for other countries (see Supplementary Details, Fig. S9). Moreover, we noticed that a significant fraction $(42 \%)$ of people were not ready to take precautions like air borne diseases, though recent evidences strongly suggest it [1]. This fraction was quite low in other countries (see Supplementary Details, Fig. S9). We noted that people were overcautious about surface stability of SARS-CoV-2, the virus attributed to COVID-19. A significant fraction (36\%) of people expected SARS-CoV-2 to survive on arbitrary surfaces for more than a week, which contradicts with reported results [3, 34]. A similar pattern was also observed for other countries (see Supplementary Details, Fig. S9). This might indicate a possible reason of panic among the people.

(A)

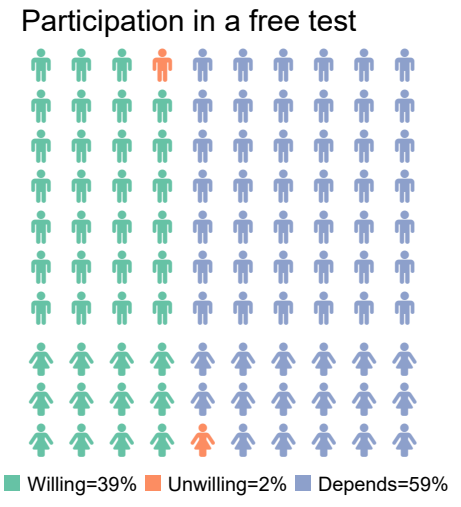

(B)

Air borne disease like precaution

舟 舟

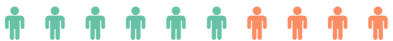

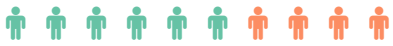

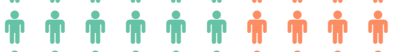

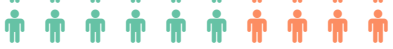

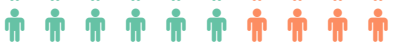

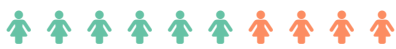

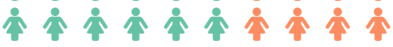

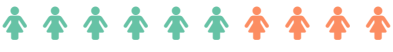
nequired $=58 \%$ not required $=42 \%$
(C) SARS-CoV-2 remains on surfaces

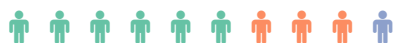

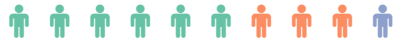

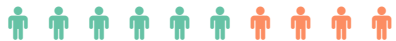

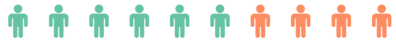

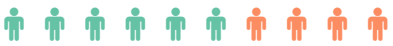

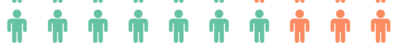

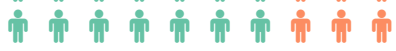

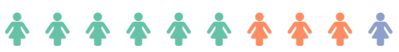

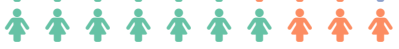

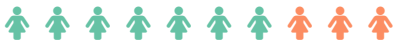
Days $=64 \%$ Weeks $=33 \%$ Months $=3 \%$

Figure 9: Gender-specific cautiousness about COVID-19 of the respondents in India. (A) Waffle plot depicting willingness to participate in free test offered by the Government for COVID-19. The conditional willingness depends on either having relevant symptoms or physical contact with someone infected. (B) Waffle plot depicting whether precautions are taken like air borne diseases. (C) Waffle plot depicting perception about survival of SARS-CoV-2, the virus responsible for COVID-19, on any arbitrary surfaces.

As hashtags depict the theme or specific content of a post on social media [33], analysis of popular hashtags might reveal new knowledge associated with an event. To study the popularity of contents related to COVID-19 on social media, we selected the top relevant hashtags appearing in India since the COVID-19 outbreak (see Supplementary Details, Table. S5). We found that many hashtags are confined to limited locations although more than 
200 countries got affected by COVID-19. On further examining the hashtags, we noticed many region-specific clusters of top hashtags. As for example, India and USA share a good number of top hashtags. This might be one of the reasons why many of the observations on Indian population differs from the other countries. We also noted that some of the drugs under trial for COVID-19 (azithromycin, hydroxychloroquine and remdesivir) were within the top hashtags. Such inclusions highlight an increasing discussion on them thereby generating different kind of panic in some countries. This is because these hashtags were popular within limited countries.

We performed sentiment analysis on the responses shared by people regarding the best and worst experiences they have faced during the lockdown in India (see Supplementary Details, Table S6-S11). "Reading" was identified across all the ages as an important entity of best experience. "Spending (quality) time" and "family time" appeared to be one of the most positive experiences. We found the main topics of "education" getting reflected in all categories of age up to 30 (including major school and college students). Gender-based assumed roles are not reflected much. Even though women in India reported "parents" as one of the leading best things while men mentioned "exercising". "Family" was reported as both best and worst context, even though differently for Indian male and female. Amongst Indian women, we observed the terms "panic" and "fear" being mentioned more than male counterparts. Irrespective of the opinion that social distancing is the best thing to curb the spread, it emerged to be the worst thing for many people. Looking at the education level, for people with Bachelors and Masters, "lost jobs" appeared to be the top worst thing.

Qualitative analysis of the detailed experiences revealed that people have become more attached to the family and enjoyed the activities like cooking. Reading and learning, mostly for the younger people, had also been a relishing factor (see Supplementary Details, Fig. S10). On the other hand, long spare time, confinement, anxiety, and loss of job had been the considerable worst experiences. Major suggestions that came from people to cope up with lockdown period were not related to personal activities rather restricted to the association with family. We can infer many novel things directly from the quantitative analyses on the survey responses. However, this gives a generic perspective of the reasons behind panic and its effects. To get an idea about the critical issues that might generate panic among the people, we conducted a qualitative analysis on the additional comments provided by the respondents (see Supplementary Details). It is interesting to note that, diversity of causes of panic in India is much higher than the other countries. Moreover, males are observed to be more vocal in providing additional suggestions.

We adopted a citizen science approach to bring respondents in the analysis pipeline [13]. The entire survey responses received from 1075 people and initial experimental results were shared with the respondents. Full accessibility was given to the respondents toward the study resources. To make the study reproducible, we also released the source codes of the experimental analysis on GitHub. All the respondents were requested to participate as citizen scientists for extending the analysis wherever possible to enrich the paper. Interestingly, high school students also took part in this process and received authorship by making significant contribution. New observations and significant results were included in the paper. Revisions to statistical analyses were also performed based on the feedback from citizen scientists. 


\section{Conclusion}

The cross-sectional analysis we carried out not only reveals the panic but also highlights some positive thoughts of people. Many people welcomed this pandemic with a belief that this would make doctors and researchers well prepared for future attacks and shape politicians more responsible. Many interesting ideas to avoid boredom were also proposed. These opinions require more attention to understand the mental health of people. While many people think that social media is helpful but it can also be disastrous because information-based decisions are becoming very much common now-a-days. We also received many important suggestions to improve the survey questionnaire. These include the information like the frequency of going outside for meeting the essential needs, personality traits, and lifestyle choices and their responses during a crisis. It is highly promising to conduct epidemiological studies further on a separate population that has not experienced COVID-19 containment for obtaining additional insights.

\section{Availability}

The complete questionnaire, dataset with anonymized survey responses, and codes used (to create plots and perform statistical analyses) in this paper are freely accessible from the GitHub link: https://github.com/malaybhattacharyya/COVID-19_Panic_Survey.

\section{Conflict of Interest}

The authors declare that they have no conflict of interest.

\section{Consent to Participate}

All the participants were informed about the goal of the survey before they participated and the results were shared with them for their consent before publication.

\section{Funding}

No funding to mention.

\section{Authors' Contributions}

$\mathrm{CB}, \mathrm{DC}, \mathrm{NA}$ and MB designed the survey. $\mathrm{CB}$ and $\mathrm{MB}$ prepared the plan of experimentation. $\mathrm{CB}, \mathrm{DC}$ and $\mathrm{MB}$ carried out the initial experiments and data analysis. SO, BB and SKM performed additional analyses as citizen scientists. CB, DC and MB drafted the manuscript. All the authors read and approved the final manuscript. 


\section{References}

[1] Prateek Bahl, Con Doolan, Charitha de Silva, Abrar Ahmad Chughtai, Lydia Bourouiba, and C Raina MacIntyre. Airborne or droplet precautions for health workers treating COVID-19? The Journal of Infectious Diseases, 2020.

[2] Alexandre Bovet and Hernán A Makse. Influence of fake news in Twitter during the 2016 US presidential election. Nature communications, 10(1):1-14, 2019.

[3] Alex Chin, Julie Chu, Mahen Perera, Kenrie Hui, Hui-Ling Yen, Michael Chan, Malik Peiris, and Leo Poon. Stability of SARS-CoV-2 in different environmental conditions. THE LANCET Microbe, 1(1):E10, 2020.

[4] Stanley Cohen. Folk devils and moral panics: The creation of the mods and rockers. Psychology Press, 2002.

[5] Edson Da Silva. Mental Health and Online Information During the COVID-19 Pandemic. InterAmerican Journal of Medicine and Health, 3, 2020.

[6] Anneliese Depoux, Sam Martin, Emilie Karafillakis, Raman Preet, Annelies WilderSmith, and Heidi Larson. The pandemic of social media panic travels faster than the COVID-19 outbreak. Journal of Travel Medicine, 2020.

[7] Jennifer Beam Dowd, Liliana Andriano, David M Brazel, Valentina Rotondi, Per Block, Xuejie Ding, Yan Liu, and Melinda C Mills. Demographic science aids in understanding the spread and fatality rates of COVID-19. Proceedings of the National Academy of Sciences, 117(18):9696-9698, 2020.

[8] Deena Dimple Dsouza, Shalini Quadros, Zainab Juzer Hyderabadwala, and MA Mamun. Aggregated COVID-19 suicide incidences in India: Fear of COVID-19 infection is the prominent causative factor. 2020.

[9] Luca Flesia, Valentina Fietta, Elena Colicino, Barbara Segatto, and Merylin Monaro. Stable psychological traits predict perceived stress related to the COVID-19 outbreak. 2020 .

[10] Laurie Garrett. COVID-19: the medium is the message. The Lancet, 395(10228):942943, 2020.

[11] Joshua Geleris, Yifei Sun, Jonathan Platt, Jason Zucker, Matthew Baldwin, George Hripcsak, Angelena Labella, Daniel Manson, Christine Kubin, R Graham Barr, et al. Observational study of hydroxychloroquine in hospitalized patients with covid-19. New England Journal of Medicine, 2020.

[12] Imran Ijaz Haider, Farah Tiwana, and Sania Mumtaz Tahir. Impact of the COVID-19 Pandemic on Adult Mental Health. Pakistan Journal of Medical Sciences, 36(COVID19S4), 2020. 
[13] Eric Hand. People power: networks of human minds are taking citizen science to a new level. Nature, 466(7307):685-688, 2010.

[14] Solomon Hsiang, Daniel Allen, Sebastien Annan-Phan, Kendon Bell, Ian Bolliger, Trinetta Chong, Hannah Druckenmiller, Andrew Hultgren, Luna Yue Huang, Emma Krasovich, et al. The effect of large-scale anti-contagion policies on the coronavirus (covid-19) pandemic. medRxiv, 2020.

[15] Abhay B Kadam and Sachin R Atre. Social media panic and COVID-19 in India. Journal of Travel Medicine, 2020.

[16] Wolfram Kawohl and Carlos Nordt. COVID-19, unemployment, and suicide. The Lancet Psychiatry, 7(5):389-390, 2020.

[17] Alfred HJ Kim, Jeffrey A Sparks, Jean W Liew, Michael S Putman, Francis Berenbaum, Alí Duarte-García, Elizabeth R Graef, Peter Korsten, Sebastian E Sattui, Emily Sirotich, et al. A rush to judgment? Rapid reporting and dissemination of results and its consequences regarding the use of hydroxychloroquine for COVID-19, 2020.

[18] Chei Sian Lee and Long Ma. News sharing in social media: The effect of gratifications and prior experience. Computers in Human Behavior, 28(2):331-339, 2012.

[19] Chi Chiu Leung, Tai Hing Lam, and Kar Keung Cheng. Mass masking in the COVID-19 epidemic: people need guidance. Lancet, 395(10228):945, 2020.

[20] Robert G Maunder, Molyn Leszcz, Diane Savage, Mary Anne Adam, Nathalie Peladeau, Donna Romano, Marci Rose, and Rabbi Bernard Schulman. Applying the lessons of SARS to pandemic influenza. Canadian Journal of Public Health, 99(6):486-488, 2008.

[21] Mandeep R Mehra, Sapan S Desai, Frank Ruschitzka, and Amit N Patel. Hydroxychloroquine or chloroquine with or without a macrolide for treatment of covid-19: a multinational registry analysis. The Lancet, 2020.

[22] G Miller. Researchers are tracking another pandemic, too-of coronavirus misinformation, 2020. March 24.

[23] Michael Y Ni, Lin Yang, Candi MC Leung, Na Li, Xiaoxin I Yao, Yishan Wang, Gabriel M Leung, Benjamin J Cowling, and Qiuyan Liao. Mental Health, Risk Factors, and Social Media Use During the COVID-19 Epidemic and Cordon Sanitaire Among the Community and Health Professionals in Wuhan, China: Cross-Sectional Survey. JMIR Mental Health, 7(5):e19009, 2020.

[24] Maria Nicola, Zaid Alsafi, Catrin Sohrabi, Ahmed Kerwan, Ahmed Al-Jabir, Christos Iosifidis, Maliha Agha, and Riaz Agha. The socio-economic implications of the coronavirus and COVID-19 pandemic: A review. International Journal of Surgery, 2020.

[25] World Health Organization et al. Mental health and psychosocial considerations during the COVID-19 outbreak, 18 March 2020. Technical report, World Health Organization, 2020. 
[26] Felipe Ornell, Jaqueline B Schuch, Anne O Sordi, and Felix Henrique Paim Kessler. "Pandemic fear" and COVID-19: mental health burden and strategies. Brazilian Journal of Psychiatry, (AHEAD), 2020.

[27] Hans Rosenberg, Shahbaz Syed, and Salim Rezaie. The Twitter pandemic: the critical role of Twitter in the dissemination of medical information and misinformation during the COVID-19 pandemic. Canadian Journal of Emergency Medicine, pages 1-7, 2020.

[28] Deblina Roy, Sarvodaya Tripathy, Sujita Kumar Kar, Nivedita Sharma, Sudhir Kumar Verma, and Vikas Kaushal. Study of knowledge, attitude, anxiety \& perceived mental healthcare need in Indian population during COVID-19 pandemic. Asian Journal of Psychiatry, page 102083, 2020.

[29] Kaushal Shah, Dhwani Kamrai, Hema Mekala, Birinder Mann, Krishna Desai, and Rikinkumar S Patel. Focus on mental health during the coronavirus (COVID-19) pandemic: applying learnings from the past outbreaks. Cureus, 12(3), 2020.

[30] Leo Sher. COVID-19, anxiety, sleep disturbances and suicide. Sleep Medicine, 2020.

[31] Kai Shu, Amy Sliva, Suhang Wang, Jiliang Tang, and Huan Liu. Fake news detection on social media: A data mining perspective. ACM SIGKDD Explorations Newsletter, 19(1):22-36, 2017.

[32] Kang Sim, Hong Choon Chua, Eduard Vieta, and George Fernandez. The anatomy of panic buying related to the current COVID-19 pandemic. Psychiatry Research, 2020.

[33] Tamara A Small. What the hashtag? A content analysis of Canadian politics on Twitter. Information, Communication \& Society, 14(6):872-895, 2011.

[34] Neeltje Van Doremalen, Trenton Bushmaker, Dylan H Morris, Myndi G Holbrook, Amandine Gamble, Brandi N Williamson, Azaibi Tamin, Jennifer L Harcourt, Natalie J Thornburg, Susan I Gerber, et al. Aerosol and surface stability of SARS-CoV-2 as compared with SARS-CoV-1. New England Journal of Medicine, 382(16):1564-1567, 2020 .

[35] Przemyslaw M Waszak, Wioleta Kasprzycka-Waszak, and Alicja Kubanek. The spread of medical fake news in social media-the pilot quantitative study. Health Policy and Technology, 7(2):115-118, 2018.

[36] Hyekyung Woo, Youngtae Cho, Eunyoung Shim, Kihwang Lee, and Gilyoung Song. Public trauma after the Sewol Ferry disaster: the role of social media in understanding the public mood. International Journal of Environmental Research and Public Health, 12(9):10974-10983, 2015.

[37] Yu-Tao Xiang, Yuan Yang, Wen Li, Ling Zhang, Qinge Zhang, Teris Cheung, and Chee H Ng. Timely mental health care for the 2019 novel coronavirus outbreak is urgently needed. The Lancet Psychiatry, 7(3):228-229, 2020. 
[38] Hao Yao, Jian-Hua Chen, and Yi-Feng Xu. Patients with mental health disorders in the COVID-19 epidemic. The Lancet Psychiatry, 7(4):e21, 2020.

[39] Stephen X Zhang, Yifei Wang, Asghar Afshar Jahanshahi, and Valentina Gomes Haensel Schmitt. First study on mental distress in Brazil during the COVID-19 crisis. medRxiv, 2020. 